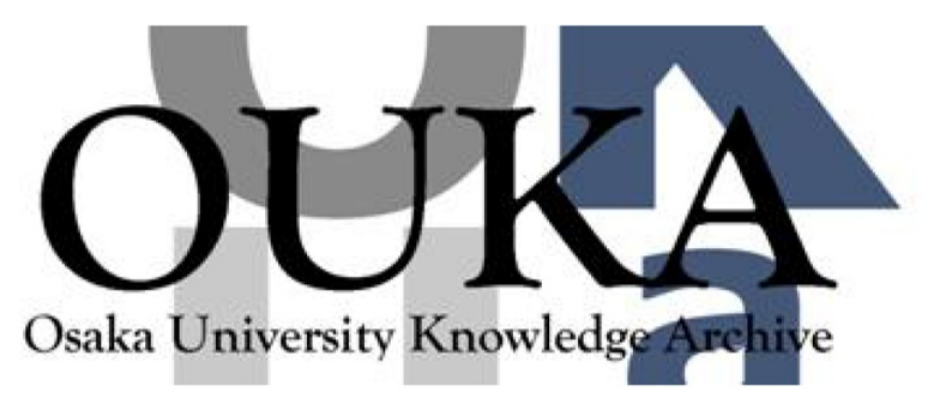

\begin{tabular}{|c|l|}
\hline Title & $\begin{array}{l}\text { Performance Evaluation of an Advanced DWDM } \\
\text { RoFSO System for Transmitting Multiple RF } \\
\text { Signals }\end{array}$ \\
\hline Author(s) & $\begin{array}{l}\text { Bekkali, Abde lmoula; Dat, PhamTien; Kazaura, } \\
\text { Kamugisha et al. }\end{array}$ \\
\hline Citation & $\begin{array}{l}\text { IEICE Transact ions on Fundamentals of } \\
\text { Electronics, Commun icat ions and Computer } \\
\text { Sciences. E92-A(11) p. 2697-p. 2705 }\end{array}$ \\
\hline Issue Date & $2009-11$ \\
\hline oaire:version & VoR \\
\hline URL & https://hdl. handle. net/11094/3030 \\
\hline rights & copyright $\odot 2008$ IEICE \\
\hline Note & \\
\hline
\end{tabular}

Osaka University Knowledge Archive : OUKA

https://ir. Library. osaka-u. ac. jp/

Osaka University 


\title{
Performance Evaluation of an Advanced DWDM RoFSO System for Transmitting Multiple RF Signals
}

\author{
Abdelmoula BEKKALI ${ }^{\dagger a}$, Pham Tien DAT $^{\dagger}$, Student Members, $^{\text {Kamugisha KAZAURA }}{ }^{\dagger}$, \\ Kazuhiko WAKAMORI ${ }^{\dagger}$, Mitsuji MATSUMOTO ${ }^{\dagger}$, Takeshi HIGASHINO ${ }^{\dagger \dagger}$, \\ Katsutoshi TSUKAMOTO ${ }^{\dagger \dagger}$, Members, and Shozo KOMAKI ${ }^{\dagger \dagger}$, Nonmember
}

\begin{abstract}
SUMMARY With the increase of communication demand and the emergence of new services, various innovative wireless technologies have been deployed recently. Free Space Optics (FSO) links combined with Radio over Fiber $(\mathrm{RoF})$ technology can realize a cost-effective heterogeneous wireless access system for both urban and rural areas. In this paper, we introduce a newly developed advanced DWDM Radio-on-FSO (RoFSO) system capable of simultaneously transmitting multiple Radio Frequency (RF) signals carrying various wireless services including W-CDMA, WLAN IEEE802.11g and ISDB-T signals over FSO link. We present an experimental performance evaluation of transmitting RF signals using the RoFSO system over a $1 \mathrm{~km}$ link under different deployment environment conditions. This work represents a pioneering attempt, based on a realistic operational scenario, aiming at demonstrating the RoFSO system can be conveniently used as a reliable alternative broadband wireless technology for complementing optical fiber networks in areas where the deployment of optical fiber is not feasible.

key words: Radio on Free Space Optics (RoFSO), Radio over Fiber (RoF),
\end{abstract} W-CDMA, ISDB-T, WLAN

\section{Introduction}

Transmission of RF signals by means of optical fiber links, referred to as Radio over Fiber ( $\mathrm{RoF})$, has been utilized for many years to facilitate wireless access. In RoF implementation, to distribute the RF signals from central station to remote stations, RF signals are placed on optical carriers and transmitted over high capacity optical fiber cables. The advantages of RoF include distributing RF signals at large bandwidth, immunity to radio frequency interference, low attenuation loss and dynamic resource allocation [1], [2]. However, RoF is dependent on the availability and cost of the installed optical fiber cables.

Free Space Optics (FSO) is the technology in which modulated optical signal is propagated over free space without using optical fiber medium. The advantages of FSO communications include ease of deployment, license free operation, high transmission security, high bit rates, full duplex transmission and protocol transparency. Depending on deployment scenario and application [3], [4]. FSO links can conveniently be used to transmit RF signals, referred to as

\footnotetext{
Manuscript received March 9, 2009.

Manuscript revised June 8, 2009.

tThe authors are with the Graduate School of Global Information and Telecommunication Studies/Institute (GITS/GITI), Waseda University, Tokyo, 169-0051 Japan.

${ }^{\dagger}$ The authors are with the Departement of Electrical, Electronic and Information Engineering, Graduate School of Engineering, Osaka University, Suita-shi, 565-0871 Japan.

a)E-mail: bekkali@fuji.waseda.jp

DOI: 10.1587/transfun.E92.A.2697
}

Radio-on-Free Space Optics (RoFSO), where the physical connection by the means of optical fiber cables is impractical, due to high costs or other considerations, with similar capacity of optical fiber [5]. The performance of RoFSO link can be degraded by many effects, such as fog, rain, atmospheric turbulence and the non-ideal characteristics of optical transmitters and receivers. To achieve high quality RF signals reception over the RoFSO system the link has to be engineered to perform in environments affected by atmospheric turbulence, low visibility, rain, building sway, etc.

Recently researches investigating the transmission of RF signals using FSO links have been reported in [6], [7]. In [6], investigation of simultaneous transmission of multiple analog RF signals over a FSO link spanning $3 \mathrm{~m}$ using WDM technology is presented. In this setup the used antenna does not utilize any tracking function because of the short distance and real operational environment characteristics are not reflected. Whereas in [7], transmission of single cellular signal using conventional FSO systems operating at $810 \mathrm{~nm}$ and $1550 \mathrm{~nm}$ over a $500 \mathrm{~m}$ link is investigated. However, in these researches, the authors did not consider the influence of atmosphere which is considered as the limited factor to FSO communication on the performance of transmitted RF signals over FSO links. Apart from the above-mentioned published work, so far the authors are not aware of any significant attempt to measure, characterize and quantify the transmission of RF signals in RoFSO implementations taking into account actual deployment environment characteristics.

In this paper we present results obtained from experimental demonstration of the newly developed advanced DWDM RoFSO system capable of simultaneously transmitting multiple wireless services including W-CDMA, WLAN IEEE802.11g and terrestrial digital broadcasting TV (ISDBT) signals over a $1 \mathrm{~km}$ link. This work represents a more realistic operational scenario with the aim of demonstrating long term system performance under different deployment environment conditions.

This paper is organized as follows. In Sect. 2, we describe the design concept and operation characteristics of the DWDM RoFSO system. Sections 3 and 4 present the experimental setup and the transmission results and analysis of W-CDMA, WLAN and ISDB-T signals using the RoFSO link. Finally, Sect. 5 concludes the paper and gives directions for future work. 


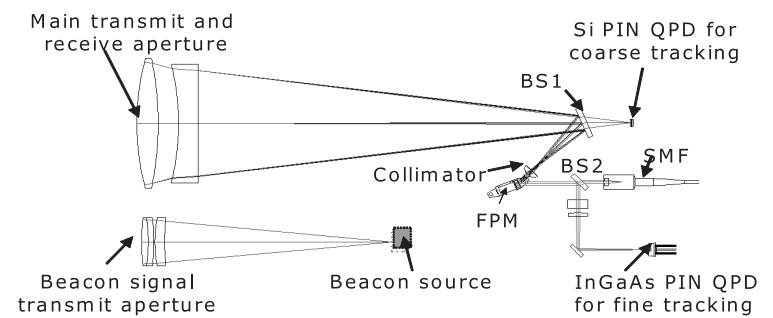

Fig. 1 DWDM RoFSO optical path and devices layout.

\section{RoFSO System Design Concept}

The next generation FSO communication systems use the $1550 \mathrm{~nm}$ wavelength in order to overcome the bandwidth and power limitation associated with the conventional FSO systems [8]. In these systems, a significant performance enhancement is achieved by using technology that has been successfully utilized to give a major boost to fiber transmission capacity like EDFA and WDM [4]. In the next generation FSO systems, an optical beam is emitted directly from a fiber termination point to free-space using a FSO transceiver and at the receiver, the transmitted optical beam is focused directly to a fiber core using the receiver optics in the receiving antenna. In this configuration, the necessity of converting the signal from electrical to optical and vice versa for transmitting or receiving through free-space is eliminated which results in a bandwidth and protocol transparent communication link. This important characteristics makes the next generation FSO system a suitable candidate for implementing the proposed advanced DWDM RoFSO system we have developed [9], [10] and also makes it readily compatible with existing widely deployed access networks based on passive optical networks (PON) [11], [12].

The basic transceiver components, design concept and operating characteristics of the advanced DWDM RoFSO system is similar to the next generation FSO system with the only difference being that the RoFSO system is optimized for transporting multiple RF signals using optical carriers by RoF technology. By using DWDM technology, multiple RF signals simultaneously transported using this system. Detailed design features and operation of the new DWDM RoFSO system have been reported in [10], [13]. Figure 1 shows the optical path layout and receiver optics configuration in the advanced DWDM RoFSO antenna.

Regardless of its obvious advantages, the performance of RoFSO systems is faced with challenges due to the extreme weather-dependency of the RoFSO channel and unlike RF based wireless systems, RoFSO links are restricted to stringent line-of-sight requirements. In order to transport as much optical power as possible to the opposite receiver the beam is usually narrow (a few tens of $\mu \mathrm{rad}$ ), the narrow transmission beam of a free-space optical signal makes alignment of RoFSO terminals difficult as compared to the wider beam RF systems. Therefore tracking and acquisition is one of the important design issues of RoFSO based com-
Table 1 Specifications of the advanced DWDM RoFSO antenna.

\begin{tabular}{|l|l|}
\hline Parameter & Specification \\
\hline \hline Operating wavelength band & $1550 \mathrm{~nm}$ \\
\hline Transmit power & $100 \mathrm{~mW}(20 \mathrm{dBm})$ \\
\hline Antenna aperture & $80 \mathrm{~mm}$ \\
\hline Coupling losses & $5 \mathrm{~dB}$ \\
\hline Beam divergence & $\pm 47.3 \mu \mathrm{rad}$ \\
\hline Fiber coupling technique & Direct coupling using FPM \\
\hline Tracking method & $\begin{array}{l}\text { Automatic using QPD } \\
\text { Rough: } 850 \mathrm{~nm} \text { beacon } \\
\end{array}$ \\
& Fine: $1550 \mathrm{~nm}$ \\
\hline
\end{tabular}

FPM: Fine Pointing Mirror used to control and steering the optical beam to single mode fiber (SMF) core

munication systems. A slight mispointing of such narrow beam could cause a complete interruption of the communication link. Numerous methods for coarse and fine tracking as well as automatic acquisition have been developed and are applied in practical FSO systems [3].

The advanced DWDM RoFSO antenna uses an innovative technique for initial antenna tracking and alignment and a technique for directly coupling the received optical beam to the single mode fiber (SMF) core i.e. fine tracking. For initial alignment the RoFSO antenna uses auto tracking whereby the beam is automatically realigned toward the opposite receiver. It incorporates a mechanism that detects the position of the beam at the receiver side using a Si PIN QPD and a counter measure that controls and keeps the beam on the receiver aperture. A beacon beam $(850 \mathrm{~nm})$ that is separate from the data carrying beam $(1550 \mathrm{~nm})$ is used for this coarse tracking and initial alignment. The automatic tracking feature uses a close loop feedback control mechanism to keep the receive beam on target.

For coupling the received optical beam to the SMF i.e. the fine tracking process, the advanced DWDM RoFSO transceiver utilizes another quadrant photo detector (InGaAs PIN QPD) and a fine pointing mirror (FPM). The information on the arrival beam position changes is provided by the QPD in order for the FPM to counteract the changes and always lead the horizontal optical axis to the fiber connection port. A digital signal processor (DSP) performs the actual position detection and analysis and feeds the information back to the fine tracking system for counter measures such as moving the FPM mirror actuators. The FPM tracking speed is chosen to be able to suppress the effects of random beam angle-of-arrival (AOA) fluctuations as a result of atmospheric turbulences on the received beam and controls and focus most of the received optical beam to the $8-10 \mu \mathrm{m}$ core of the SMF. The specification of the DWDM RoFSO system used in the experiments described in this paper is given in Table 1.

\section{RoFSO System Transmission Characteristics}

\subsection{Experimental Setup}

To evaluate the transmission performance of the developed advanced DWDM RoFSO system, we setup up two RoFSO 


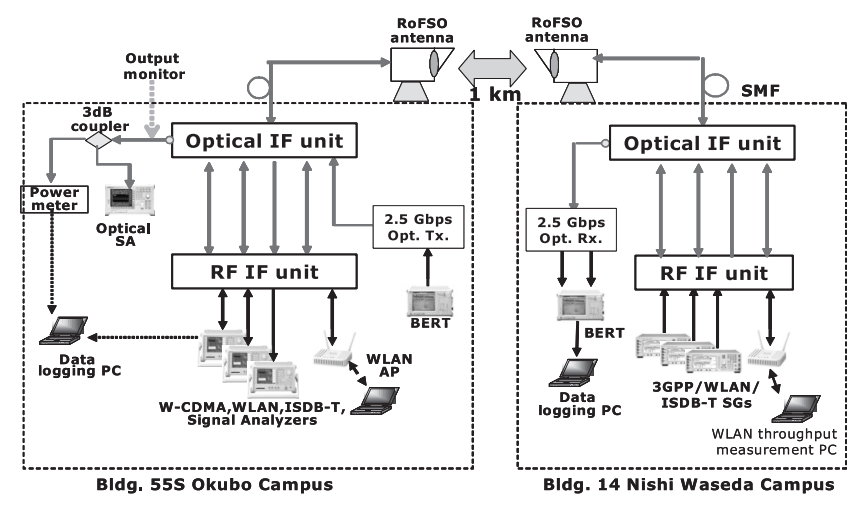

Fig. 2 Experimental setup for evaluation of the DWDM RoFSO system.

antennas on the rooftop of two buildings in Waseda University campus area in Tokyo city. The two buildings are separated by a distance of $1 \mathrm{~km}$ which is within the range of typical application distance for FSO links [4], [14]. A schematic diagram representing the experimental setup of the RoFSO system is shown in Fig. 2. Placed at one site are signal generators for producing different kind of wireless service signals which are then multiplexed together and transmitted via the DWDM RoFSO link by the RoFSO antenna placed on the buildings rooftop. At the second site, signal analyzers and other devices for measuring and recording the quality of the received RF and optical signals, weather data (temperature, visibility, rainrate etc) as well as atmospheric conditions like scintillation effects are placed.

In the RoFSO system configuration two interface units are included; an optical interface unit (Optical IF unit) and a RoF interface unit (RF IF unit). The optical interface unit consists of a wavelength multiplex and de-multiplex device, boost and post amplifier and an optical circulator to isolate the transmit and received signals. On the other hand, the RoF interface unit has RoF modules responsible for the electrical to optical signal conversion and vice versa corresponding to each wireless service RF signal under investigation.

The success of all FSO links depends on a optimized link budget. In this experimental investigation the FSO link was designed and engineered to provide enough link margin to compensate for losses. However, The link margin is dependent on the requirement of the FSO link availability, and the kind of wireless services to be transmitted over RoFSO link. The $1 \mathrm{Km}$ RoFSO link parameters are given in Table 2 . The link parameters include the optical losses inherent in the RoFSO system devices as well as the light loss during propagation. In order to describe the FSO link losses due to the atmospheric turbulence, rain, low visibility and tracking loss, several models were published in the literature [3], [4].

In our experimental setup and inverstigation the test signals under consideration include standard digital signal at $2.5 \mathrm{Gbps}$, cellular signals (3GPP W-CDMA signal) at $2 \mathrm{GHz}$, WLAN signal (IEEE $802.11 \mathrm{~g} / \mathrm{a}$ at $2.4 \mathrm{GHz}$ and $5 \mathrm{GHz}$ respectively) as well as digital terrestrial television broadcasting (ISDB-T) signal at the UHF band [470 MHz, $770 \mathrm{MHz}$; all transmitted simultaneously using DWDM
Table $21 \mathrm{~km}$ RoFSO link parameters.

\begin{tabular}{|l|l|}
\hline Parameter & Value \\
\hline \hline Booster EDFA output & $+20 \mathrm{dBm}$ \\
\hline Tx./Rx. WDM MUX/DEMUX loss & $1.5 \mathrm{~dB}$ \\
\hline Tx./Rx. Optical circulator loss & $1 \mathrm{~dB}$ \\
\hline Tx./Rx. Antenna coupling loss & $5 \mathrm{~dB}$ \\
\hline Fiber cable and connectors losses & $3 \mathrm{~dB}$ \\
\hline FSO geometrical loss [4] & $4 \mathrm{~dB}$ \\
\hline Post EDFA gain & $+25 \mathrm{dBm}$ \\
\hline
\end{tabular}

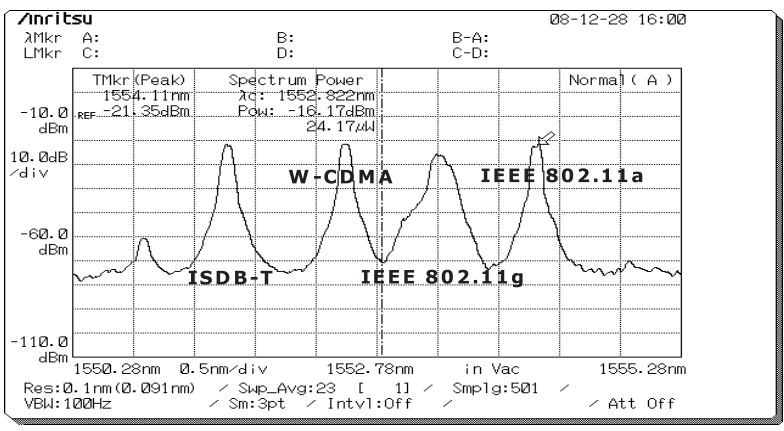

Fig. 3 Received WDM spectrum.

Table 3 Wireless signal wavelength assignment.

\begin{tabular}{|l|c|l|l|}
\hline & Ch.\# & Wavelength & Wireless service \\
\hline \hline \multirow{3}{*}{ Downlink } & 29 & $1554.13 \mathrm{~nm}$ & WLAN IEEE802.11a \\
\cline { 2 - 4 } & 30 & $1553.33 \mathrm{~nm}$ & WLAN IEEE802.11g \\
\cline { 2 - 4 } & 31 & $1552.52 \mathrm{~nm}$ & Cellular W-CDMA \\
\cline { 2 - 4 } & 32 & $1551.72 \mathrm{~nm}$ & ISBD-T \\
\hline \hline \multirow{3}{*}{ Uplink } & 33 & $1550.92 \mathrm{~nm}$ & Free \\
\cline { 2 - 4 } & 34 & $1550.12 \mathrm{~nm}$ & Cellular W-CDMA \\
\cline { 2 - 4 } & 35 & $1549.32 \mathrm{~nm}$ & WLAN IEEE802.11g \\
\cline { 2 - 4 } & 36 & $1548.52 \mathrm{~nm}$ & WLAN IEEE802.11a \\
\hline
\end{tabular}

technology over the RoFSO link. We evaluate the DWDM RoFSO system performance by measuring and analyzing the quality of the RF signals transmitted over it based on the quality metric parameters specified in the standards for transmission of the different kind of wireless services.

Figure 3 depicts the received optical signal WDM spectrum showing from left to right: the ISDB-T, W-CDMA, $802.11 \mathrm{~g}$ and $802.11 \mathrm{a}$ signals carrying wavelengths. The WDM wavelengths are separated using the ITU-T Recommendation G.692 $100 \mathrm{GHz}$ grid spacing [15]. Except for the $802.11 \mathrm{~g}$ wavelength, the rest of the WDM signals show ideal characteristics because they are generated using signal generators which produce signals waveforms with almost ideal characteristics. The $802.11 \mathrm{~g}$ signal is slightly different because it is an actual WLAN 802.11g access point (AP) signal connection via the RoFSO link. The received WDM spectrum depicted in Fig. 3 shows a stable performance. The wavelength assignments for the wireless services signals downlink and uplink are given in Table 3. Channel 33 which is designated as "free" and is used for evaluating the RoFSO system performance in terms of BER by transmitting a 2.5 Gbps digital signal. 


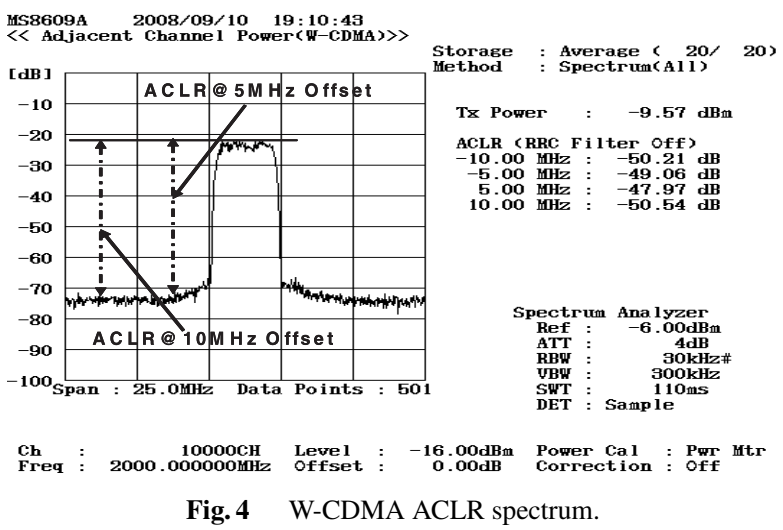

\subsection{W-CDMA Signal Transmission}

For cellular W-CDMA signal transmission experiment at $2 \mathrm{GHz}$, channel 31 is used for downlink and channel 34 is used for uplink. In W-CDMA system, the downlink signal transmitted by the base station is designed to fulfill the specifications set in 3GPP standard [16]. The spectral properties of the signal are measured by the adjacent channel leakage ratio (ACLR), considered to be a more stringent quality metric parameter, and is defined as the ratio of the amount of leakage power in an adjacent channel to the total transmitted power in the main channel. The 3GPP specifies one main channel and two adjacent channels. The standard requires the ACLR to be better than $45 \mathrm{~dB}$ at $5 \mathrm{MHz}$ offset and $50 \mathrm{~dB}$ at $10 \mathrm{MHz}$ offset.

A signal generator (Agilent E4438C) is used to generate a test signal W-CDMA Test Model 1which consists of 64 Dedicated Physical Channels (DPCH), with a spreading factor of 128, distributed randomly across the code space, at random power levels and random timing offsets so as to simulate a realistic traffic scenario that may have high PAR (Peak to Average Ratio). The signal is generated with a power of $-20 \mathrm{dBm}$ which is fed to a RoF module (in the $\mathrm{RF}$ interface unit) with an optical modulation index (OMI) of $10 \%$ and then transmitted over the RoFSO link and at the receiver side a digital mobile radio transmission tester (Anritsu MS8609A) is used to measure and record the quality of the W-CDMA signal.

Figure 4 shows a received W-CDMA signal ACLR spectrum after transmission over the $1 \mathrm{~km}$ RoFSO link. The spectral properties of the signal satisfy the 3GPP specified values of ACLR at the $5 \mathrm{MHz}$ and $10 \mathrm{MHz}$ offsets. The variation of the measured received optical power and the W-CDMA signal ACLR characteristics is shown in Fig. 5. Two cases are considered i.e. first case is back-to-back measurement (B-to-B) using the RoF modules, signal generator and analyzer and an optical attenuator (HP8156A) for incrementing the attenuation to represent channel losses and in the second case actual transmission over the RoFSO link is conducted. The ACLR is measured for both 5 and $10 \mathrm{MHz}$ offsets. The back-to-back and actual transmission over the RoFSO system measurements shows almost similar charac-

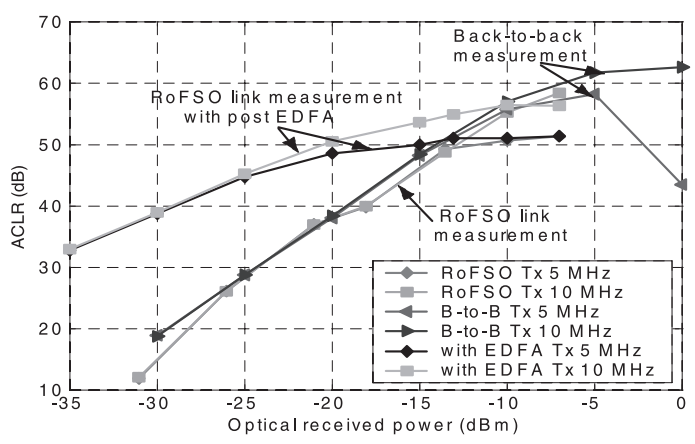

Fig. 5 Variation of ACLR with the received optical power.

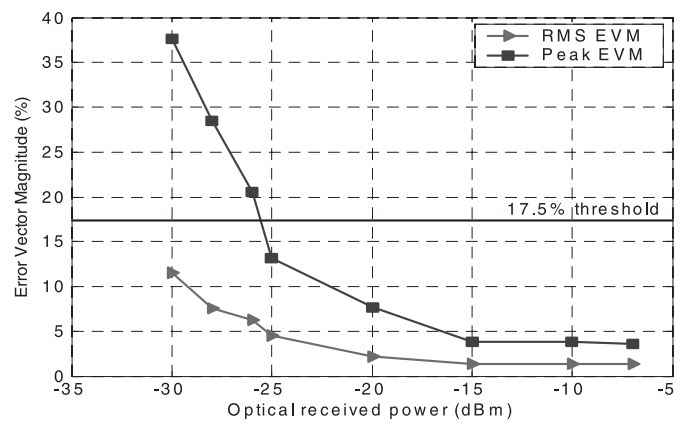

Fig. 6 Variation of EVM with the received optical power.

teristics with the minimum received optical power to satisfy the prescribed $3 \mathrm{GPP}$ specification value at $5 \mathrm{MHz}$ at $10 \mathrm{MHz}$ offsets being approximately $-15 \mathrm{dBm}$. Using a post EDFA the required received optical power can be even as low as $-25 \mathrm{dBm}$ and $-20 \mathrm{dBm}$ at $5 \mathrm{MHz}$ and $10 \mathrm{MHz}$ offsets respectively and still satisfy the $3 \mathrm{GPP}$ specification for W-CDMA signal transmission.

An Error Vector Magnitude (EVM) quality metric parameter is also used to evaluate the performance of the RoFSO system for W-CDMA signal transmission. The EVM metric, which is a ratio in percent of the difference between the reference waveform and the measured waveform, provides the measure of the modulation quality of the transmitter. The 3GPP standard requires the EVM not to exceed $17.5 \%$. The measured rms EVM characteristics after transmission over the RoFSO link for measured received optical power ranging from $-30 \mathrm{dBm}$ to $-7 \mathrm{dBm}$ is below the threshold limit set by the 3GPP standard as depicted in Fig. 6. However, the EVM is not a very stringent quality metric parameter for measurement of performance of WCDMA signal transmission when compared to ACLR.

\subsection{ISDB-T Signal Transmission}

Digital terrestrial television broadcasting, referred to as Integrated Service Digital Broadcasting - Terrestrial (ISDB-T) in Japan, is developed to provide reliable high-quality video, sound and data broadcasting not only for fixed receivers but also for mobile receivers [17]. The ISDB-T system uses the UHF band at frequencies between $470 \mathrm{MHz}$ and $770 \mathrm{MHz}$, 
Table 4 ISDB-T transmission parameters.

\begin{tabular}{|l|c|c|}
\hline Pattern name & ISDBT_16QAM_1_2 \\
\hline \hline Mode & \multicolumn{2}{|c|}{3} \\
\hline Guard Interval & \multicolumn{2}{|c|}{$1 / 8$} \\
\hline Layer & A & B \\
\hline Number of segments & 1 & 12 \\
\hline Carrier Modulation & 16 QAM & 64 QAM \\
\hline Inner Coding rate & $1 / 2$ & $7 / 8$ \\
\hline Information bit rate $[\mathrm{kbps}]$ & 624.13 & 19660 \\
\hline
\end{tabular}

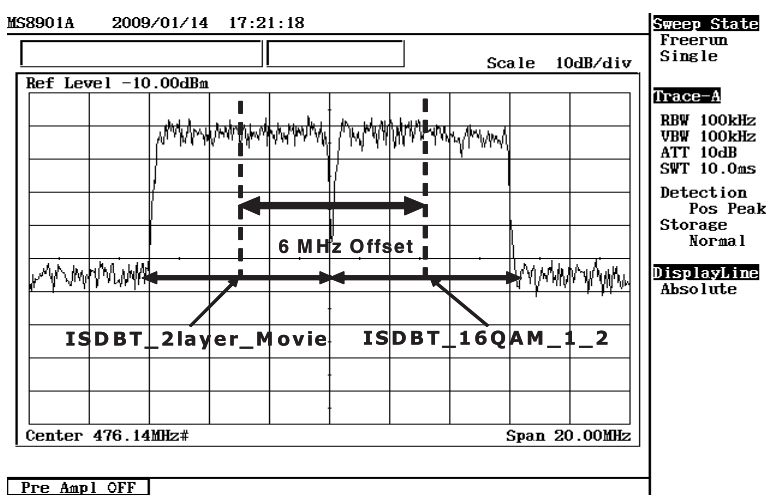

Fig. 7 ISDB-T received signal spectrum.

giving a total bandwidth of $300 \mathrm{MHz}$. The bandwidth is divided into 50 channels numbred from 13 to 62 . Each channel is further divided into 13 OFDM segments which includes a single segment, (Layer_A or 1seg) for mobile receivers (LDTV, audio and data) and the remainder can be allocated as one 12-segments (Layer_B) for high definition television (HDTV) programs [18].

In this setup, channel 32 is used for ISDB-T signal transmission. A vector signal generator (Anritsu MG3700A) is used to generate waveforms for digital terrestrial television broadcasting (ISDB-T) transmission evaluation. In this experiment, two signals are set simultaneously with the following waveform patterns (a) ISDBT_16QAM_1_2 and (b) ISDBT_2layer_Movie, both at $-20 \mathrm{dBm}$ with a $6 \mathrm{MHz}$ frequency offset. The combined signal at $-17 \mathrm{dBm}$ is fed into the RoF module. The OMI for each channel (at $-20 \mathrm{dBm}$ input) is $10 \%$. The signal is subsequently transmitted over the RoFSO link. The parameters of the two signals are listed in Table 4. At the receiving site a digital broadcasting signal analyzer (Anritsu MS8901A) is used to measure the quality of the received ISDB-T signal in terms of Bit Error Rate (BER) and Modulation Error Ratio (MER). It should be noted that, the waveform pattern (a) is mainly used for BER and MER measurement and the waveform pattern (b) is mainly used for evaluation of video and voice data terminals. A received signal spectrum showing the two transmitted ISDB-T signals is depicted in Fig. 7.

The ISDB-T signal transmission using the RoFSO system is first evaluated using a MER quality metric parameter. The MER is a measure used to quantify the performance of a digital radio transmitter or receiver in a communications system using digital modulation. It gives an indication of the

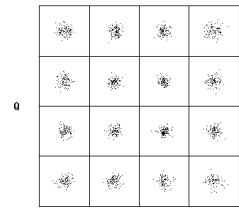

(a)

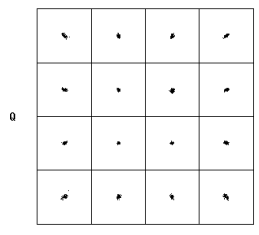

(c)

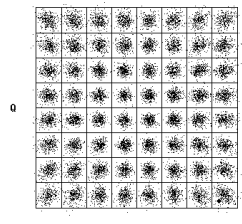

(b)

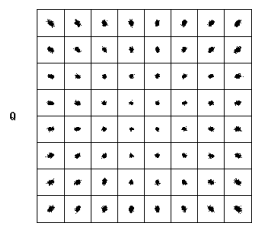

(d)
Fig. 8 ISDB-T constellation map (a) 1 seg (MER $=23 \mathrm{~dB}$ ), (b) $12 \mathrm{seg}$ $($ MER $=23 \mathrm{~dB}),(\mathrm{c}) 1 \mathrm{seg}($ MER $=35 \mathrm{~dB}),(\mathrm{d}) 12 \mathrm{seg}($ MER $=35 \mathrm{~dB})$.


Fig. 9 Received ISDB-T MER characteristics.

ability of the receiver to correctly decode the signal [19]. In the RoF link the measured MER will be influenced by both $\mathrm{RF}$ noise figure and intermodulation distortion (IMD).

An example of modulation analysis constellation for the ISDB-T signal made of Layer_A (16QAM) and Layer_B (64QAM) for different values of MER is shown in Figs. 8(a), (b), (c) and (d) respectively. The constellation is very useful for analyzing the condition of the received signal by monitoring the modulation symbol movement.

Figure 9 depicts a long term data of the received ISDBT MER measured from 15th February 2009 at $04 \mathrm{~h} 00$ to 16th February 2009 at $04 \mathrm{~h} 00$. The data collected every $1 \mathrm{sec}-$ ond and averaged every 1 minute in clear weather condition with a visibility between $10 \mathrm{~km}$ and $20 \mathrm{~km}$. The visibility is a useful measure of the atmosphere containing fog, dust, smoke and other contaminating particles. It characterizes the transparency of the atmosphere estimated by the distance at which an object or light can be clearly discerned. It can be observed that the value of MER is almost higher than $11.5 \mathrm{~dB}$ for Layer_A and $22 \mathrm{~dB}$ for Layer_B, satisfying the requirement for ISDB-T signal transmission [20]. However, the received MER drops and shows a fast fluctuation with the changes of the received optical power. This is due to the 


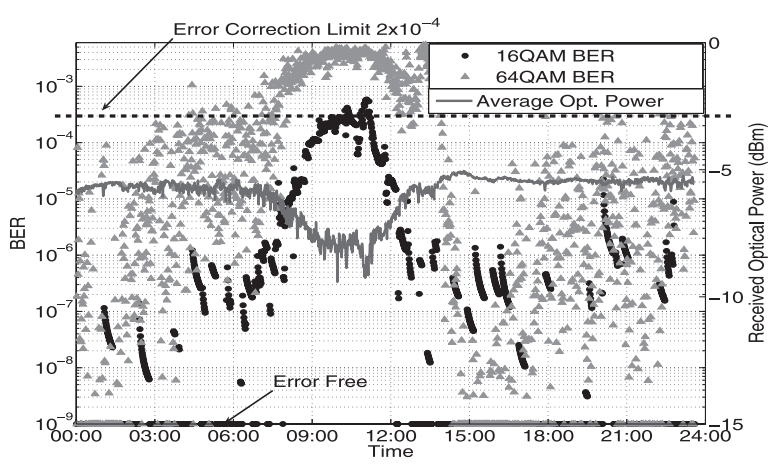

Fig. 10 Received ISDB-T BER characteristics.

atmospheric turbulence which is attributed to the fact that during the day the solar radiation warms up the ground and air causing irradiance fluctuations (known as scintillation) in the received optical signals propagating along an horizontal path near ground [14], [21]. The structure constant of refractive index fluctuations $\mathrm{C}_{n}^{2}$, is the parameter most commonly used to describe the strength of atmospheric turbulence. $\mathrm{C}_{n}^{2}$ is governed by the fluctuation of the refractive index of air and can vary from $10^{-13}\left(\mathrm{~m}^{-2 / 3}\right)$ for strong turbulence to $10^{-16}\left(\mathrm{~m}^{-2 / 3}\right)$ for weak turbulence. From Fig. 9 , it can be observed that the MER variance increases with the attenuation of the received optical power during the strong atmospheric turbulence period $\left(\mathrm{C}_{n}^{2}>5 \times 10^{-14}\left(\mathrm{~m}^{-2 / 3}\right)\right)$. In clear weather condition, the atmospheric loss is due to the scintillation variance and can be expressed by Eq. (1) as function of $\mathrm{C}_{n}^{2}\left(\mathrm{~m}^{-2 / 3}\right), \lambda(\mathrm{m})$ the transmitter wavelength and $l(m)$ the transmission link distance [14]. Atmospheric turbulence remains the major issue to establish a reliable RoFSO link operating over long distances.

$$
\operatorname{Att}_{\text {Scintillation }}[\mathrm{dB}]=2 \sqrt{23.17\left(\frac{2 \pi}{\lambda}\right)^{7 / 6} C_{n}^{2} l^{11 / 6}}
$$

In this experiment we also measured the ISDB-T signal BER quality metric parameter. The ISDB-T BER must be less than $2 \times 10^{-4}$ after the inner-code correction (Viterbi decoding) and before the outer code correction RS $(204,188)$ [20]. Results of the received ISDB-T Layer_A and Layer_B BER and received optical power characteristics for a clear day are shown in Fig. 10. The data is recorded for a continuous 24 hour of 11th December 2008 and the average received optical power represents the average value of received optical power recorded in 1 minute interval. Similar to the case of the MER, the Layer_A BER, Layer_B BER and the received optical power characteristics are observed to deteriorate causing increased burst errors especially at noon time. These errors are attributed to the increase in the atmospheric turbulence induced beam wander and scintillation effects which are usually stronger around midday, resulting in random attenuation of the received signal.

The BER characteristics show satisfactory performance with most values being below the error correction limit $\left(2 \times 10^{-4}\right)$. Unfortunately, the automatic gain con-

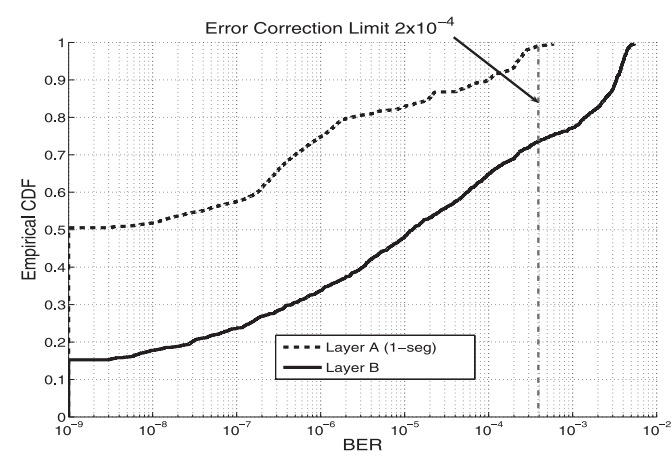

Fig. 11 Empirical CDF of ISDB-T BER.

trol (AGC) is occasionally inadequate in the case of 12segments (Layer_B) transmission. However, the system achieves better performance where error free transmission can be observed. To get better insight of this data, the emperical cumulative distribution function (CDF) of Layer_A and Layer_B BER is plotted in Fig. 11. It is shown that most likely $99 \%$ of Layer_A BER data is better than the required value of $2 \times 10^{-4}$ with $50 \%$ of the data are error free, while for the Layer_B BER $72 \%$ of the recorded data is under the threshold with $18 \%$ error free. Therefore, under moderate atmospheric turbulence period, the system shows satisfactory BER performance for both Layer_A (16QAM) and Layer_B (64QAM) which are better than the standard requirement, demonstrating the suitability of the RoFSO system for ISDB-T signal transmission.

In this paper, the main focus is on the discussion of the transmission of ISDB-T signal using wireless optical link as wireless optical relay between a central station (CS) and a remote distribution station (RDS) by exploiting the benefits of RoF and FSO technologies. However, the performance of the RoFSO system in ISDB-T broadcasting service area is not considered here.

\subsection{IEEE 802.11g Signal Transmission Results}

Using another vector signal generator (MG3700A), an IEEE $802.11 \mathrm{~g}$ compliant signal waveform pattern is generated and after transmission through the RoFSO link a spectrum analyzer (Anritsu MS2687B) is utilized to measure and analyze the quality of the received WLAN signals. For the IEEE $802.11 \mathrm{~g}$ experiment the signal was generated at $-20 \mathrm{dBm}$ and fed into the RoF module with a OMI of $10 \%$. The WLAN signal transmission is evaluated using the quality metric parameters specified by [22], [24] standard specifications.

A Pass/Fail judgment of the spectrum mask as defined in the IEEE specification $802.11 \mathrm{~g}$ is used [22]. The spectrum mask defines the permitted distribution of power across each channel from the 14 channels allocated for WLAN in Japan. The mask requires that the signal should be attenuated by at least $20 \mathrm{~dB}$ from its peak energy at $\pm 11 \mathrm{MHz}$ 


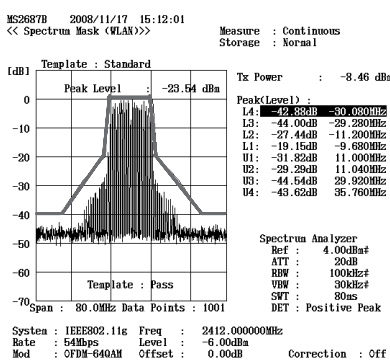

(a)

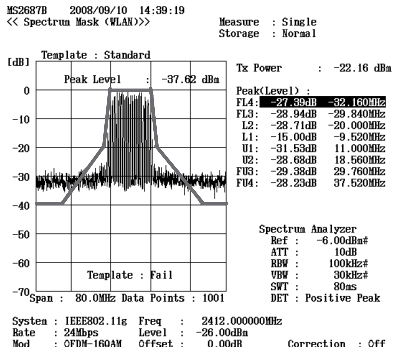

(b)
Fig. 12 WLAN802.11g spectrum mask (a) Pass, (b) Fail.

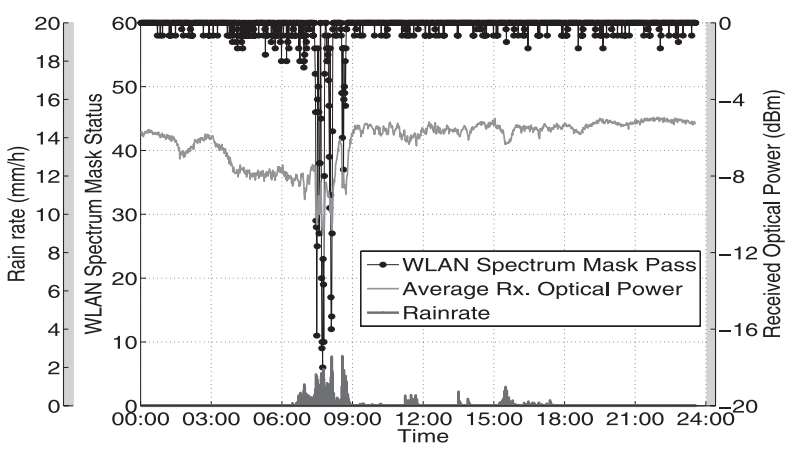

Fig. 13 WLAN 802.11g spectrum mask status characteristics.

from the centre frequency, the sense in which channels are effectively $22 \mathrm{MHz}$ wide. An example of the spectrum mask test of WLAN IEEE802.11g waveform at $2.4 \mathrm{GHz}$ with $54 \mathrm{Mbps}$, 64QAM is shown in Figs. 12(a) and (b). In the example shown the IEEE $802.11 \mathrm{~g}$ signal spectrum mask test passes (Fig. 12(a)) i.e. is in compliance with the specified standard while for Fig. 12(b) the spectrum mask test fails. Here, the failure to meet the specified standards is due to the received optical power attenuation caused by the rainfall i.e. the increase of the amplified spontaneous emission (ASE) noise generated in the post EDFA. In general, the spectrum mask failure is due to the out-band additive noise, which is dominated by the beat noise between the signal and the ASE (Signal-ASE) at the post EDFA, and the LD intermodulation distortion power near the desired spectrum.

Figure 13 shows the variation of the number in which the spectrum mask test of WLAN passed within 1 minute for a data collected in 1 second intervals in continuous 24 hours of 16th November 2008. The maximum number of Pass/Fail times is 60 for each minute. In the graph, the received optical power represents the average value of received optical power recorded in 1 minute interval. A sudden drop of the number of WLAN spectrum mask Pass characteristics can be observed with the drop of the received optical power while the remainder of the days collected data showed Pass most of the time. This is due to the little rainfall which was recorded (about $3 \mathrm{~mm} / \mathrm{hr}$ ) and the weather became slight overcast which could explain the increased of spectrum mask fail number and drops in the optical received power. Usually in rainy days, the temperature remains al-

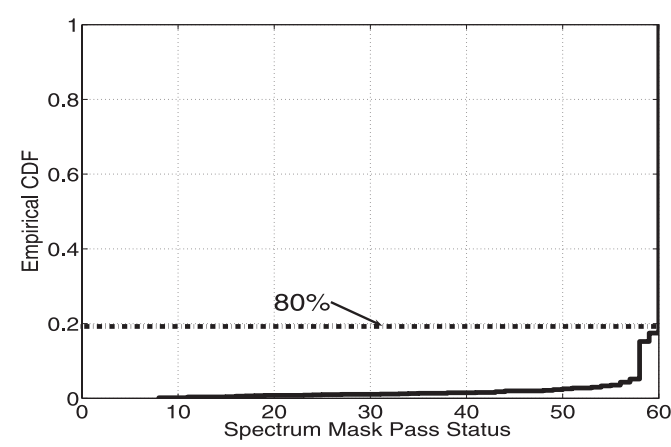

Fig. 14 CDF of WLAN $802.11 \mathrm{~g}$ spectrum mask status.

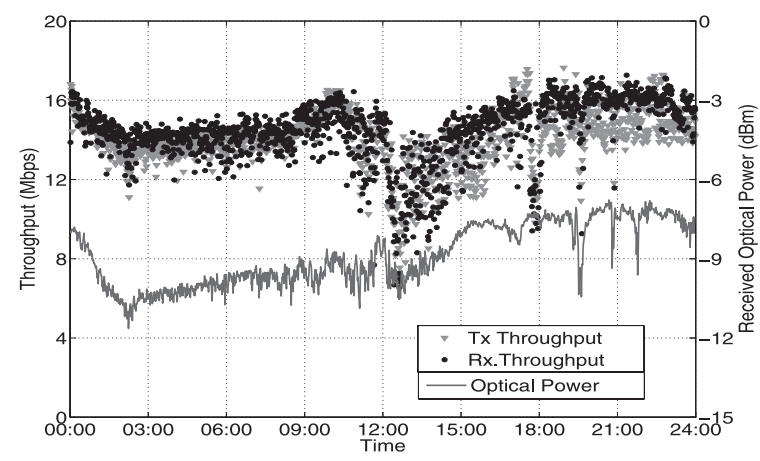

Fig. 15 WLAN 802.11g measured throughput characteristics.

most constant so the effect of temperature changes on the optical beam is not significant and thus the effect of atmospheric turbulence can be ignored. In this case, rainfall and the low visibility are the major sources of the laser beam attenuation, which can be compensated by increasing the signal gain. The attenuation due to the rain can be expressed as function of Rainrate $R[\mathrm{~mm} / \mathrm{h}]$ by [23]

$$
\operatorname{Att}_{\text {Rain }}[\mathrm{dB} / \mathrm{km}]=1.076 R^{2 / 3}
$$

Figure 14 shows the plot of the CDF of spectrum mask pass/fail status for data recorded in a continuous 15 days from 18th November 2008 to 3rd December 2008, with different weather conditions clear and rainy. We can see from the CDF plot that $80 \%$ of data exhibits spectrum mask pass in a continuous 60 seconds with $99 \%$ of data is Pass $50 \%$ of times in one minute.

Two computers placed at the two sites and connected via a WLAN (IEEE 802.11g) access point (AP) to the RoFSO link is conducted. A throughput measurement application is utilized to transmit continuously a $64 \mathrm{MB}$ file size in $8 \mathrm{~KB}$ packet size chunks. The throughput data is collected continuously ( 24 hours) in clear weather day. Figure 15 illustrates the variation of the throughput characteristics with respect to the measured average received optical power. In this particular measurement, the throughput is consistently above $12 \mathrm{Mbps}$ and seems to drop below this value only around midday a time which is usually characterized with increased transmission errors because of atmospheric turbulence induced scintillation etc. This result con- 
firms that the RoFSO system is stable and can provide consistent TCP/IP data transmission even when the link quality deteriorates due to atmospheric turbulence.

\section{Conclusion}

An experimental evaluation on simultaneous transmission of different kinds of wireless service signals using a newly developed advanced DWDM RoFSO system under real operational environment has been presented. Important performance metric parameters for evaluating the quality of WCDMA, ISDB-T and WLAN IEEE $802.11 \mathrm{~g} / \mathrm{a}$ signals transmission using RoFSO link have been measured and characterized under various atmospheric influences and weather conditions.

From the initial results on the performance evaluation of the RoFSO system we have confirmed that for W-CDMA signal transmission the minimum required received optical power should be $-15 \mathrm{dBm}$ without post EDFA and $-20 \mathrm{dBm}$ when using post EDFA. In the case of ISDB-T Layer_A and Layer_B signal transmission the BER measured data showed a CDF of $99 \%$ and $72 \%$ respectively of the values appearing below the specified error correction limit. Finally for the WLAN $802.11 \mathrm{~g}$ transmission the CDF of the spectrum mask measurement showed 99\% successful pass. These results confirm the technical feasibility and practicality of utilizing the RoFSO system as a universal platform for providing ubiquitous wireless services.

Future work involves investigation on performance analysis of the RoFSO system in the service area, the improvement in the tracking mechanism and performance improvement of the different RoF modules utilized for each $\mathrm{RF}$ wireless service. Extensive measurements will be conducted to obtain further experimentally derived models for a better understanding of the system performance.

\section{Acknowledgment}

This work is supported by a grant from the National Institute of Information and Communications Technology (NiCT) of Japan.

\section{References}

[1] H. Al-Raweshidy and S. Komaki, eds., Radio over Fiber Technologies for Mobile Communications Networks, first ed., Artech House Publishers, 2002.

[2] W.S.C. Chang, ed., RF Photonic Technology in Optical Fiber Links, 1st ed., Cambridge Univ. Press, 2002.

[3] H. Willebrand and B. Ghuman, Free Space Optics: Enabling Optical Connectivity in Today's Networks, Sams Publishing, 2002.

[4] S. Bloom, E. Korevaar, J. Schuster, and H. Willebrand, "Understanding the performance of free-space optics," Optical Networking, vol.2, pp.178-200, June 2003.

[5] H. Refai, J. Sluss, and M. Atiquzzaman, "A comparative study of the performance of analog fiber-optic links versus free-space optical links," Optical Engineering, vol.45, no.2, pp.025003.1-10, Feb. 2006.

[6] H. Refai, J. Sluss, and H. Refai, "The transmission of multiple RF signals in free-space optics using wavelength division multiplexing," Atmospheric Propagation II, vol.5793, SPIE, May 2005.

[7] G. Katz, S. Arnon, P. Goldgeier, Y. Hauptman, and N. Atias, "Cellular over optical wireless networks," IEE Proc. Optoelectron, vol.153, pp.195-198, Aug. 2006.

[8] I.I. Kim, M. Mitchel, and E. Korevaar, "Measurement of scintillation for free-space laser communication at $785 \mathrm{~nm}$ and $1550 \mathrm{~nm}$," Optical Wireless Communications II, vol.3850, pp.49-62, SPIE, Sept. 1999.

[9] K. Kazaura, K. Omae, T. Suzuki, M. Matsumoto, E. Mutafungwa, T. Murakami, K. Takahashi, H. Matsumoto, K. Wakamori, and Y. Arimoto, "Performance evaluation of next generation free-space optical communication system," IEICE Trans. Electron., vol.E90-C, no.2, pp.381-388, Feb. 2007

[10] K. Tsukamoto, K. Nakaduka, M. Kamei, T. Higashino, S. Komaki, K. Wakamori, Y. Aburakawa, T. Nakamura, K. Takahashi, T. Suzuki, K. Kazaura, K. Omae, M. Matsumoto, S. Kuwano, and H. Watanabe, "Development of DWDM radio on free space optic link system for ubiquitous wireless," AP-MWP Conference, April 2007.

[11] ITU-T Rec. G.983.1, "Broadband optical access systems based on passive optical networks (PON)," Jan. 2005.

[12] F. Effenberger, D. Cleary, O. Haran, G. Kramer, R.D. Li, M. Oron, and T. Pfeiffer, "An introduction to PON technologies," IEEE Commun. Mag., vol.45, pp.S17-S25, March 2007.

[13] K. Takahashi, T. Higashino, T. Nakamura, Y. Aburakawa, K Tsukamoto, S. Komaki, K. Wakamori, T. Suzuki, K. Kazaura, A.M Shah, K. Omae, M. Matsumoto, and Y. Miyamoto, "Design and evaluation of optical antenna module suitable for radio-on free-space optics link system for ubiquitous wireless," Free-Space Laser Communication Technologies XX, vol.6877, SPIE, Jan. 2008.

[14] L.C. Andrews and R.L. Phillips, Laser Beam Propagation Through Random Media, 2nd ed., SPIE-International Society for Optical Engineering, 2005.

[15] ITU-T Rec. G.692, "Optical interfaces for multichannel systems with optical amplifiers," Oct. 1998.

[16] 3GPP TS 25.141, http://www.3gpp.org/, 2002.

[17] DiBEG, http://www.dibeg.org/

[18] ARIB STD-B31, "Transmission system for digital terrestrial television broadcasting."

[19] ETSI Technical Report, ETR 290, "Measurement guidelines for digital video broadcasting (DVB) systems."

[20] ARIB STD-B21, "Receiver for digital broadcasting."

[21] H. Weichel, Laser Beam Propagation in the Atmosphere, first ed., SPIE Optical Engineering Press, 1990.

[22] http://standards.ieee.org/getieee802/802.11.html

[23] T.H. Carbonneau and D.R. Wiseley, "Opportunities and challenges for optical wireless," SPIE on Optic. Wireless Com., vol.3232, 1998.

[24] ARIB STD-T71, "Broadband mobile access communication system (CSMA)."

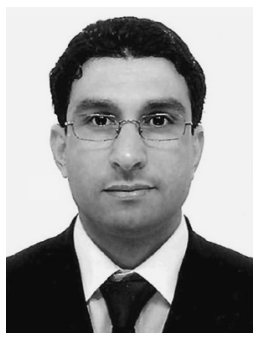

Abdelmoula Bekkali received the B.Sc. degree in Applied Mathematics and M.Sc. degree in satellite communication from Ecole Mohamedia d'Ingenieurs (EMI), Morocco in 2001. He began his career as Research and Development engineer in Radar Communication. $\mathrm{He}$ then received his M.Sc. degree in wireless communication from Waseda University's Graduate School of Global Information and Telecommunication Studies in 2007. He is currently working towards his Ph.D. degree at the same university. His research interests include Free Space Optics, Radio over Fiber, optical networks, Sensor networks and RFID. He is student member of IEEE and SPIE. 


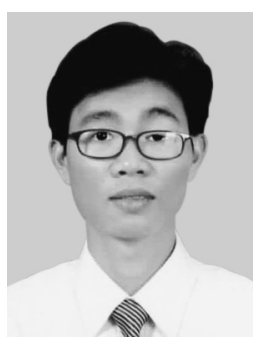

Pham Tien Dat received B.E. degree in Electronics and Telecommunication Engineering from Posts and Telecommunications Institute of Technology (PTIT), Vietnam in 2003. From 2003 to 2006, he worked as a researcher at Research Institute of Posts and Telecommunications (RIPT), Vietnam. He later joined Waseda University and received his M.Sc. in Telecommunication Engineering in 2008. He is currently working towards his Ph.D. degree in the same University. His research interests include optical networks, performance of free-space optical links and system modeling.

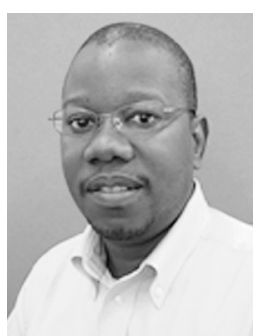

Kamugisha Kazaura received the B.Eng. (Hons) degree in Electronics and Communications Engineering from the University of Bath, in U.K., in 1995. He received the M.Sc. and Ph.D. degrees in Global Information and Telecommunication Studies from Waseda University's Graduate School of Global Information and Telecommunication Studies in 2002 and 2007 respectively. Prior to joining Waseda University he worked as an Executive Engineer for the Tanzania Telecommunications Company Ltd. (TTCL) between 1996 and 2002. Since 2008, he has been a visiting lecturer with the Research Institute for Science and Engineering of Waseda University and a principal investigator in various projects. His research interests lie in the general field of fixed and mobile high-speed wireless communications networks, communication network design and free-space optics systems.

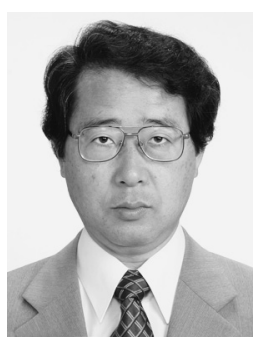

Kazuhiko Wakamori received the M.Sc. from Shizuoka University, Graduate School of Information Engineering and Ph.D. degree from Osaka City University. From 1979 he joined Hamamatsu Photonics K.K. He has been a visiting researcher at the Global Information and Telecommunication Institute of Waseda University from 2004. His research interests include development of optical communication for a high-speed optical device and research and development of free- space optical communica-

tion.

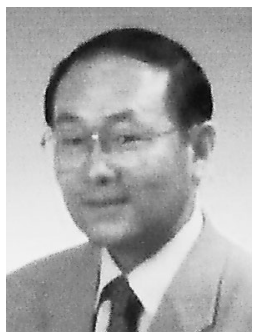

Mitsuji Matsumoto Since joining NTT labs in 1970, Dr. Matsumoto has been engaged in research and CCITT standardization activities in the field of protocol architecture and terminal design for facsimile, telematics and multimedia services and systems. He joined the Global Information and Telecommunication Institute of Waseda University, Tokyo, Japan as professor in 1996. Currently he is vice director of GITI. In 2000-2004 study period he became Vice Chairman of ITU-T SG16 (Multimedia) and since 2004 he is the vice president of Infrared Data Association.

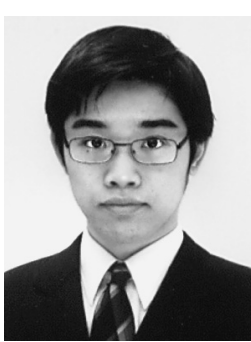

Takeshi Higashino was born in Osaka, Japan in November 11, 1978. He received the B.E., M.E. and Ph.D. degrees in Communications Engineering from Osaka University, in 2001, 2002 and 2005 respectively. He is currently a Research Associate in the Department of Electrical, Electronic and Information Engineering at Osaka University, engaging in the research on radio and optical communication systems.

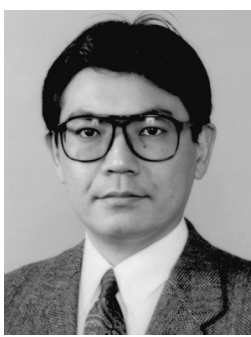

Katsutoshi Tsukamoto was born in Shiga, Japan in October 7, 1959. He received the B.E., M.E. and Ph.D. degrees in Communications Engineering from Osaka University, in 1982, 1984 and 1995 respectively. He is currently an Associate Professor in the Department of Communications Engineering at Osaka University, engaging in the research on radio and optical communication systems. He is a member of IEEE and ITE. He was awarded the Paper Award of IEICE, Japan in 1996.

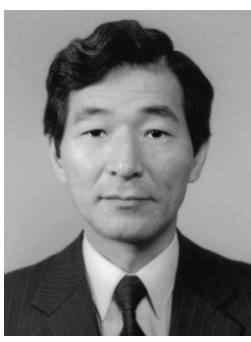

Shozo Komaki was born in Osaka, Japan, in 1947. He received B.E., M.E. and Ph.D. degrees in Electrical Communication Engineering from Osaka University, in 1970, 1972 and 1983 respectively. In 1972, he joined the NTT Radio Communication Labs., where he was engaged in repeater development for a $20-\mathrm{GHz}$ digital radio system, 16-QAM and 256-QAM systems. From 1990, he moved to Osaka University, Faculty of Engineering, and engaging in the research on radio and optical communication systems. He is currently a Professor of Osaka University. Dr. Komaki is a senior member of IEEE, and a member of the Institute of Television Engineers of Japan (ITE). He was awarded the Paper Award and the Achievement Award of IEICE, Japan in 1977 and 1994 respectively. 